PROCEEDINGS OF THE

AMERICAN MATHEMATICAL SOCIETY

Volume 137, Number 2, February 2009, Pages 723-731

S 0002-9939(08)09642-1

Article electronically published on September 9, 2008

\title{
ON AMALGAMATIONS OF HEEGAARD SPLITTINGS WITH HIGH DISTANCE
}

\author{
GUOQIU YANG AND FENGCHUN LEI
}

(Communicated by Daniel Ruberman)

\begin{abstract}
Let $M$ be a compact, orientable 3-manifold and $F$ an essential closed surface which cuts $M$ into $M_{1}$ and $M_{2}$. Suppose that $M_{i}$ has a Heegaard splitting $V_{i} \cup_{S_{i}} W_{i}$ with distance $D\left(S_{i}\right) \geqslant 2 g\left(M_{i}\right)+1, i=1,2$. Then $g(M)=$ $g\left(M_{1}\right)+g\left(M_{2}\right)-g(F)$, and the amalgamation of $V_{1} \cup_{S_{1}} W_{1}$ and $V_{2} \cup_{S_{2}} W_{2}$ is the unique minimal Heegaard splitting of $M$ up to isotopy.
\end{abstract}

\section{INTRODUCTION}

Let $M_{i}$ be a connected, compact, orientable 3-manifold, $F_{i}$ an essential boundary component of $M_{i}$ with $g\left(F_{i}\right) \geqslant 1, i=1,2$, and $F_{1} \cong F_{2}$. Let $\varphi: F_{1} \rightarrow F_{2}$ be a homeomorphism, and $M=M_{1} \cup_{\varphi} M_{2}$. Suppose $V_{i} \cup_{S_{i}} W_{i}$ is a Heegaard splitting of $M_{i}(i=1,2)$. Then $V_{1} \cup_{S_{1}} W_{1}$ and $V_{2} \cup_{S_{2}} W_{2}$ induce a natural Heegaard splitting $V \cup_{S} W$ of $M$ with $g(S)=g\left(S_{1}\right)+g\left(S_{2}\right)-g(F)$, which is called the amalgamation of $V_{1} \cup_{S_{1}} W_{1}$ and $V_{2} \cup_{S_{2}} W_{2}$ along $F_{1}$ and $F_{2}$. Clearly, $g(M) \leqslant g\left(M_{1}\right)+g\left(M_{2}\right)-g(F)$.

There exist examples which show that an amalgamation of two minimal genus Heegaard splittings of $M_{1}$ and $M_{2}$ is stabilized (refer to [1, 8], etc.). On the other hand, it has been shown that under some conditions on the manifolds and the gluing maps, the equality $g(M)=g\left(M_{1}\right)+g\left(M_{2}\right)-g(F)$ holds; see [10, 11, [17, etc.

The concept of Hempel's Heegaard distance of a Heegaard splitting ([5]) is a natural generalization of the concept of Casson-Gordon's weakly reducible Heegaard splitting ( 3 ); its relations to the genus of the Heegaard splitting have been discussed in [4, 6], 14], etc. For a Heegaard splitting $V \cup_{S} W$, we use $D(S)$ to denote the Heegaard distance of $V \cup_{S} W$.

Recently, Kobayashi and Qiu (9]) proved the following theorem:

Theorem 1.0. Let $M$ be a connected, compact, orientable 3-manifold, and $F$ an essential closed surface which cuts $M$ into two 3-manifolds $M_{1}$ and $M_{2}$. Suppose that $M_{i}$ has a Heegaard splitting $V_{i} \cup_{S_{i}} W_{i}$ with $D\left(S_{i}\right) \geqslant 2\left(g\left(M_{1}\right)+g\left(M_{2}\right)-g(F)\right)$, $i=1,2$. Then $M$ has a unique minimal Heegaard splitting up to isotopy, i.e. the amalgamation of $V_{1} \cup_{S_{1}} W_{1}$ and $V_{2} \cup_{S_{2}} W_{2}$.

The main result of this paper is as follows:

Received by the editors August 6, 2007.

2000 Mathematics Subject Classification. Primary 57M99.

Key words and phrases. Amalgamation, distance of Heegaard splitting, minimal Heegaard splitting.

The second author is supported in part by a grant (No. 15071034) of NFSC and a grant (No. 893322) of DLUT.

(C)2008 American Mathematical Society Reverts to public domain 28 years from publication 
Theorem 1.1. Let $M_{i}$ be a connected, compact, orientable 3-manifold, $F_{i}$ an essential boundary component of $M_{i}$ with $g\left(F_{i}\right) \geqslant 1, i=1,2$, and $F_{1} \cong F_{2}$. Let $\varphi: F_{1} \rightarrow F_{2}$ be a homeomorphism, and $M=M_{1} \cup_{\varphi} M_{2}, F=F_{2}=\varphi\left(F_{1}\right)$. Suppose $M_{i}$ has a Heegaard splitting $V_{i} \cup_{S_{i}} W_{i}$ with $D\left(S_{i}\right) \geqslant 2 g\left(M_{i}\right)+1, i=1,2$. Then the amalgamation of $V_{1} \cup_{S_{1}} W_{1}$ and $V_{2} \cup_{S_{2}} W_{2}$ is the unique minimal Heegaard splitting of $M$ up to isotopy. In particular, it is unstabilized.

As a direct consequence of Theorem 1.1 we have

Corollary 1.2. Under the conditions as in Theorem 1.1, the minimal Heegaard splitting of $M$ is weakly reducible.

The paper is organized as follows. In section 2, we introduce some preliminaries, lemmas and propositions. The main part of section 2 is to prove Proposition 2.5, which is a stronger version of Lemma 3.3 in [2. In section 3, we first prove some results that will be used in the proof of Theorem 1.1. and then give a proof of Theorem 1.1, where Proposition 2.5 plays a key role in our proofs.

The concepts and terminologies which are not defined in the paper are standard; see, for example, [5], 7].

\section{Preliminaries}

In this section, we will review some fundamental definitions and facts on surfaces in 3-manifolds.

Let $F$ be either a properly embedded connected surface in a 3-manifold $M$ or a subsurface of $\partial M$. If there is an essential curve in $F$ which bounds a disk in $M$ or $F$ is a 2 -sphere which bounds a 3 -ball in $M$, then we say $F$ is compressible in $M$. Otherwise, $F$ is incompressible in $M$. If $F$ is an incompressible surface in $M$ and not parallel to a subsurface of $\partial M$, then $F$ is an essential surface in $M$. When $F$ is not connected, then $F$ is said to be incompressible if each component of $F$ is incompressible. $F$ is said to be essential if $F$ is incompressible and at least one component of $F$ is essential in $M$.

Let $F$ be a properly embedded connected surface in a 3-manifold $M$. If there is an essential arc $\alpha$ in $F$ and an arc $\beta$ in $\partial M$ such that $\alpha \cap \beta=\partial \alpha=\partial \beta$ and $\alpha \cup \beta$ bounds a disk $\Delta$ in $M$, then $F$ is said to be $\partial$-compressible in $M$.

A compression body is a 3-manifold $V$ obtained from a connected closed orientable surface $S$ by attaching some 2-handles to $S \times\{0\} \subset S \times I$ and capping off any resulting 2 -sphere boundary components. We denote $S \times\{1\}$ by $\partial_{+} V$ and $\partial V-\partial_{+} V$ by $\partial_{-} V$. An essential disk for $V$ is a compressing disk of $\partial_{+} V$ in $V$.

A Heegaard splitting of a 3-manifold $M$ is a decomposition $M=V \cup_{S} W$ of $M$ in which $V$ and $W$ are compression bodies such that $V \cap W=\partial_{+} V=\partial_{+} W=S$ and $M=V \cup W . S$ is called a Heegaard surface of $M$. The genus $g(S)$ of $S$ is called the genus of the splitting $V \cup_{S} W$. We use $g(M)$ to denote the Heegaard genus of $M$, which is equal to the minimal genus of all Heegaard splittings of $M$. A Heegaard splitting $V \cup_{S} W$ for $M$ is minimal if $g(S)=g(M)$.

Let $V \cup_{S} W$ be a Heegaard splitting. $V \cup_{S} W$ is reducible (weakly reducible, or stabilized, respectively) if there are essential disks $D_{1} \subset V$ and $D_{2} \subset W$ such that $\partial D_{1}=\partial D_{2}\left(\partial D_{1} \cap \partial D_{2}=\emptyset\right.$, or $\left|\partial D_{1} \cap \partial D_{2}\right|=1$, respectively). Otherwise, $V \cup_{S} W$ is irreducible (strongly irreducible, unstabilized, respectively).

A generalized Heegaard splitting for a 3 -manifold $M$ is a structure $M=$ $\left(V_{1} \cup_{S_{1}} W_{1}\right) \cup_{F_{1}}\left(V_{2} \cup_{S_{2}} W_{2}\right) \cup_{F_{2}} \cdots \cup_{F_{m-1}}\left(V_{m} \cup_{S_{m}} W_{m}\right)$, where each $V_{i} \cup_{S_{i}} W_{i}$ is 
a Heegaard splitting, and $\left\{M_{i}=V_{i} \cup_{S_{i}} W_{i}, 1 \leqslant i \leqslant m\right\}$ is a union of submanifolds of $M$.

It was shown by Scharlemann and Thompson [12 that any irreducible Heegaard splitting $M=V \cup_{S} W$ can be broken up into a series of strongly irreducible Heegaard splittings by rearranging the order of adding the 1-handles and 2-handles as

$$
M=V \cup_{S} W=\left(V_{1} \cup_{S_{1}} W_{1}\right) \cup_{F_{1}}\left(V_{2} \cup_{S_{2}} W_{2}\right) \cup_{F_{2}} \cdots \cup_{F_{m-1}}\left(V_{m} \cup_{S_{m}} W_{m}\right),
$$

such that each $V_{i} \cup_{S_{i}} W_{i}$ is a strongly irreducible Heegaard splitting with $\partial_{-} W_{i} \cap$ $\partial_{-} V_{i+1}=F_{i}, 1 \leqslant i \leqslant m-1, \partial_{-} V_{1}=\partial_{-} V, \partial_{-} W_{m}=\partial_{-} W$, and for each $i$, each component of $F_{i}$ is a closed incompressible surface of positive genus, and only one component of $M_{i}=V_{i} \cup_{S_{i}} W_{i}$ is not a product, and none of the compression bodies $V_{i}, W_{i-1}, 2 \leqslant i \leqslant m$, is trivial. Such a rearrangement of handles is called an untelescoping of the Heegaard splitting $V \cup_{S} W$. Then it is easy to see $g(S) \geqslant$ $g\left(S_{i}\right), g\left(F_{i}\right)$ for each $i$, and when $m \geqslant 2, g(S)>g\left(S_{i}\right), g\left(F_{i}\right)$ for each $i$. In fact, $\chi(S)=\sum_{i=1}^{m} \chi\left(S_{i}\right)-\sum_{i=1}^{m-1} \chi\left(F_{i}\right)$.

Let $M=V \cup_{S} W$ be a Heegaard splitting, $\alpha$ and $\beta$ be two essential simple closed curves in $S$. The distance $d(\alpha, \beta)$ of $\alpha$ and $\beta$ is the smallest integer $n \geqslant 0$ such that there is a sequence of essential simple closed curves $\alpha=\alpha_{0}, \alpha_{1}, \cdots, \alpha_{n}=\beta$ in $S$ with $\alpha_{i-1} \cap \alpha_{i}=\emptyset$, for $1 \leqslant i \leqslant n$. The distance of the Heegaard splitting $V \cup_{S} W$ is defined to be $D(S)=\min \{d(\alpha, \beta)\}$, where $\alpha$ bounds an essential disk in $V$ and $\beta$ bounds an essential disk in $W$.

$D(S)$ was first defined by Hempel [6]. It is clear that $V \cup_{S} W$ is reducible if and only if $D(S)=0$, and $V \cup_{S} W$ is weakly reducible if and only if $D(S) \leqslant 1$.

Next we introduce some basic results on Heegaard splittings and the distance of a Heegaard splitting.

Lemma 2.1. Let $V$ be a compression body and $F$ be a properly embedded incompressible surface in $V$ with $\partial F \subset \partial_{+} V$. Then each component of $V \backslash F$ is a compression body.

The proof of Lemma 2.1 can be found in $[15]$.

Lemma 2.2. Let $M=V \cup_{S} W$ be a strongly irreducible Heegaard splitting. If $\alpha$ is an essential simple loop in $S$ which bounds a disk $D$ in $M$ such that $D$ is transverse to $S$, then $\alpha$ bounds an essential disk in $V$ or $W$.

The proof of Lemma 2.2 can be found in [13].

Lemma 2.3. Let $V \cup_{S} W$ be a Heegaard splitting of $M$ and $F$ be a properly embedded incompressible surface (maybe not connected) in $M$. Then any component of $F$ is parallel to $\partial M$ or $D(S) \leqslant 2-\chi(F)$.

The proof of Lemma 2.3 can be found in [4].

Lemma 2.4. Let $M=V \cup_{S} W$ and $M=V^{\prime} \cup_{S^{\prime}} W^{\prime}$ be two different Heegaard splittings. Then $V^{\prime} \cup_{S^{\prime}} W^{\prime}$ is a stabilization of $V \cup_{S} W$ or $D(S) \leqslant 2 g\left(S^{\prime}\right)$.

The proof of Lemma 2.4 can be found in 14 .

The following proposition is a stronger version of Lemma 3.3 in [2].

Proposition 2.5. Let $M=V \cup_{S} W$ be a non-trivial strongly irreducible Heegaard splitting and $F$ be a 2-sided essential surface (not a disk or 2-sphere) in $M$. Then $F$ can be isotoped such that 
(1) each component of $S \cap F$ is an essential loop in both $F$ and $S$;

(2) at most one component of $S \backslash F$ is compressible in $M \backslash F$.

Proof. (1) is due to Schultens [16].

If (2) is not true, then at least two components of $S \backslash F$ are compressible in $M \backslash F$ and by Lemma 2.2. at least two components of $S \backslash F$ are compressible in $V$ or $W$. Since $V \cup_{S} W$ is strongly irreducible, we may assume that at least two components of $S \backslash F$ are compressible in $V$ and any component of $S \backslash F$ is incompressible in $W$. Choose an essential disk $D$ of $W$ and isotope $F$ if necessary so that $|D \cap(F \cap W)|$ is minimal subject to the conditions that any component of $S \cap F$ is essential in both $F$ and $S$, and at least two components of $S \backslash F$ are compressible in $V$.

Since $V \cup_{S} W$ is strongly irreducible, $D \cap(F \cap W) \neq \emptyset$. By the standard innermost circle argument, we know that $D \cap(F \cap W)$ has no circle component. Let $\alpha$ be an outermost arc of $D \cap(F \cap W)$ in $D$ and $\triangle$ be the corresponding outermost disk. We denote $\overline{\partial \triangle-\alpha}$ by $\beta$. $\alpha$ is an essential arc in $F \cap W$ by the minimality of $|D \cap(F \cap W)|$. $\beta$ is an essential arc in $S \backslash F$, too. Otherwise, there is an arc $\gamma$ in $S \backslash F$ with $\gamma \cap \beta=\partial \gamma=\partial \beta$ and $\beta \cup \gamma$ bounds a disk $\triangle^{\prime}$. Then either $\triangle \cup \triangle^{\prime}$ is a compressing disk of $F$, a contradiction, or $\alpha \cup \gamma$ is trivial, contradicting the minimality of $|D \cap(F \cap W)|$.

If the component $P$ of $F \cap W$ containing $\alpha$ is not an annulus, then $\partial$-compress $P$ along $\triangle$ to get $F^{*}$, which is, isotopic to $F$. Any component of $F^{*} \cap S$ is essential in both $S$ and $F^{*}$. At least one component of $S \backslash F^{*}$ is compressible in $V$ since at least two components of $S \backslash F$ are compressible in $V$. If only one component of $S \backslash F^{*}$ is compressible, then Proposition 2.5 (2) is true. If at least two components of $S \backslash F^{*}$ are compressible in $V$, we have $\left|D \cap\left(F^{*} \cap W\right)\right|<|D \cap(F \cap W)|$, again a contradiction to the minimality of $|D \cap(F \cap W)|$.

Now assume $P$ is an annulus. $P$ is not parallel to any component of $S \backslash F$. Otherwise, pushing $P$ from $W$ into $V$, this corresponds to an isotopy of $F$, denoted by $F^{*}$, too. Then any component of $F^{*} \cap S$ is essential in both $S$ and $F^{*}$. At least one component of $S \backslash F^{*}$ is compressible in $V$. Then by the same argument as above, either Proposition 2.5 (2) is true or we get a contradiction.

So $P$ is an essential annulus in $W$. We $\partial$-compress $P$ along $\triangle$ to get an essential disk $E$ with $E \cap F=\emptyset$ in $W$. At least two components of $S \backslash F$ are compressible in $V$. This is a contradiction to the assumption that $V \cup_{S} W$ is strongly irreducible.

This completes the proof.

\section{The MAin Results AND PROOFS}

First, we have

Theorem 3.1. Let $M$ be a compact, orientable 3-manifold and $F$ be an essential closed surface which cuts $M$ into $M_{1}$ and $M_{2}$. If $M_{i}$ has a Heegaard splitting $V_{i} \cup_{S_{i}} W_{i}$ with $D\left(S_{i}\right) \geqslant 2 g\left(M_{i}\right)+1, i=1,2$, and $V \cup_{S} W$ is a Heegaard splitting of $M$ with $g(S) \leqslant g\left(M_{1}\right)+g\left(M_{2}\right)-g(F)$, then $V \cup_{S} W$ is weakly reducible.

Proof. Suppose $V \cup_{S} W$ is a strongly irreducible Heegaard splitting. $F$ is essential in $M$, so $F \cap S \neq \emptyset$. Then by Proposition 2.5, we may assume that $F \cap S$ consists of loops which are essential in both $F$ and $S$ and at most one component of $S \backslash F$ is compressible in $W$ or $V$, so in $M_{1}$ or $M_{2}$. With no loss of generality, we assume any component of $S \cap M_{1}$ is incompressible. Thus any component of $S \cap M_{1}$ is essential in $M_{1}$. By Lemma 2.3, $2-\chi\left(S \cap M_{1}\right) \geqslant D\left(S_{1}\right) \geqslant 2 g\left(M_{1}\right)+1$. 
By assumption, $g(S) \leqslant g\left(M_{1}\right)+g\left(M_{2}\right)-g(F)$. So

$$
\begin{gathered}
\chi\left(S \cap M_{1}\right)+\chi\left(S \cap M_{2}\right)=\chi(S)=2-2 g(S) \\
\quad \geqslant 2-2\left(g\left(M_{1}\right)+g\left(M_{2}\right)-g(F)\right) .
\end{gathered}
$$

Therefore,

$$
\begin{aligned}
-\chi\left(S \cap M_{2}\right) & \leqslant \chi\left(S \cap M_{1}\right)+2\left(g\left(M_{1}\right)+g\left(M_{2}\right)-g(F)\right)-2 \\
& \leqslant 2 g\left(M_{2}\right)-2 g(F)-1 .
\end{aligned}
$$

By Proposition 2.5, $S \cap M_{2}$ has at most one component which is compressible in $M_{2}$, and since $2-\chi\left(S \cap M_{2}\right) \leqslant 2 g\left(M_{2}\right)-2 g(F)+1<2 g\left(M_{2}\right)<D\left(S_{2}\right)$, any incompressible component of $S \cap M_{2}$ is parallel to a subsurface of $F$ in $M_{2}$.

If $S \cap M_{2}$ is incompressible, then we can isotope $F$ such that $F \cap S=\emptyset$, a contradiction. So $S \cap M_{2}$ has only one component $Q$ which is compressible in $V$ or $W$, say $V$. We compress $Q$ as much as possible in $V$, and the resulting surface is denoted by $Q^{*}$. Then $Q^{*}$ is incompressible in $M_{2}$ since $V \cup_{S} W$ is strongly irreducible. Since $2-\chi\left(Q^{*}\right) \leqslant 2-\chi\left(S \cap M_{2}\right)<D\left(S_{2}\right), Q^{*}$ is parallel to the subsurfaces of $F$; see Figure 1 .
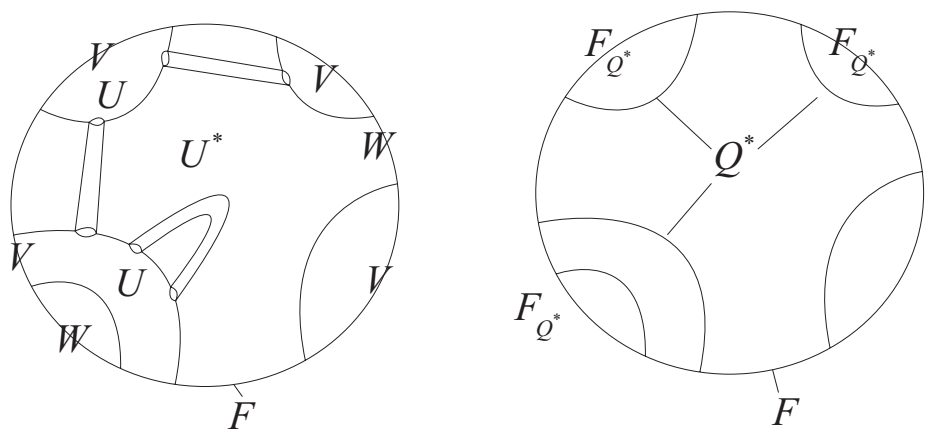

Figure 1

Obviously, any component of $F \cap V$ is incompressible in $V$ and any component of $F \cap W$ is incompressible in $W$. Then by Lemma 2.1 any component of $V \cap M_{2}$ and $W \cap M_{2}$ is a compression body. We denote the component of $V \backslash F$ which contains the component $Q$ by $U$ and the component of $W \backslash F$ which contains the component $Q$ by $U^{*}$. Then $U \cup_{Q} U^{*}$ is homeomorphic to $M_{2}$ since any incompressible component of $S \cap M_{2}$ is parallel to $F$ in $M_{2}$. For any component $A$ of $Q^{*}$, let $F_{A}$ be the subsurface of $F$ which is parallel to $A$ with $\partial A=\partial F_{A}$ and $F_{Q^{*}}=\left\{F_{A}: A \in Q^{*}\right\}$. If there are two components $A$ and $B$ of $Q^{*}$ such that $F_{A} \subseteq F_{B}$, then set $\mathcal{A}_{1}=$ $\left\{A^{\prime}: A^{\prime} \in Q^{*}, F_{A^{\prime}} \subset F_{B}, F_{A^{\prime}} \neq F_{B}\right\}$ and $\mathcal{A}_{2}=\left\{A^{\prime}: A^{\prime} \in Q^{*}, F_{A^{\prime}} \cap F_{B}=\emptyset\right\}$, and we may assume that $Q$ is compressed into $Q^{*}$ in $V$ by cutting $Q$ open along a collection $\mathcal{D}=\left\{D_{1}, \cdots, D_{n}\right\}$ of pairwise disjoint compressing disks in $V$. We claim that $\mathcal{A}_{2}=\emptyset$. Otherwise, since $Q$ is connected, there must exist $A_{1} \in \mathcal{A}_{1}$ and $A_{2} \in \mathcal{A}_{2}$, and $D_{p_{1}}, D_{p_{2}} \in \mathcal{D}$ such that the two cutting sections of $D_{p_{i}}$ lie in $A_{i}$ and $B$ respectively, $i=1,2$. But this contradicts the assumption that $Q$ is separating. So $\mathcal{A}_{2}=\emptyset$. Then $M_{2} \cong R$ is a compression body, where $R, A$ and $B$ are shown as in Figure 2 and $V_{2} \cup_{S_{2}} W_{2}$ is weakly reducible, a contradiction to the fact that $D\left(S_{2}\right) \geqslant 2 g\left(M_{2}\right)+1$. 

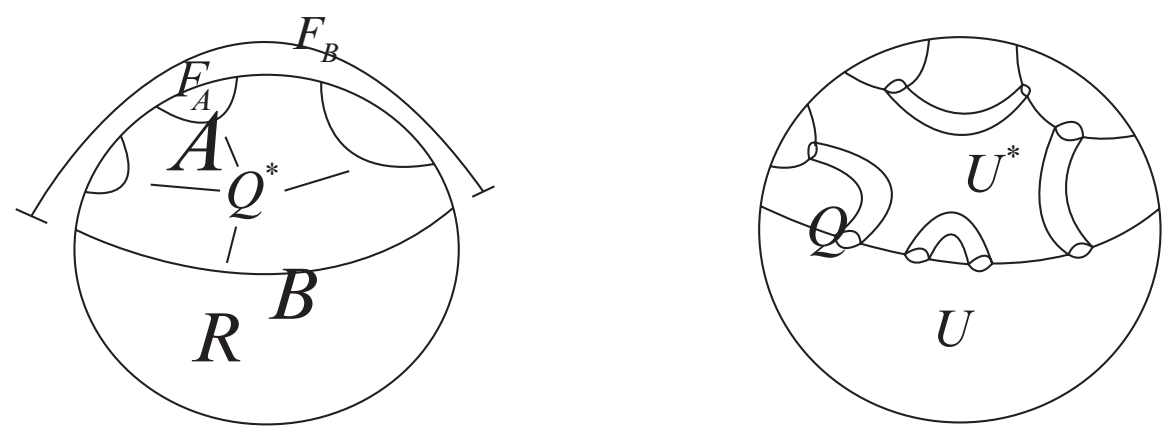

FIGURE 2

Let $C=U \cup N\left(F \cap U^{*}, U^{*}\right)$ and $C^{*}=U^{*} \backslash N\left(F \cap U^{*}, U^{*}\right)$. Then $C$ is a compression body and $C^{*}$ is a compression body with $\partial_{+} C=\partial_{+} C^{*}=S^{*}$ and $C \cup_{S^{*}} C^{*}$ is a Heegaard splitting of $U \cup_{Q} U^{*}=M_{2}$; see Figure 3 ,
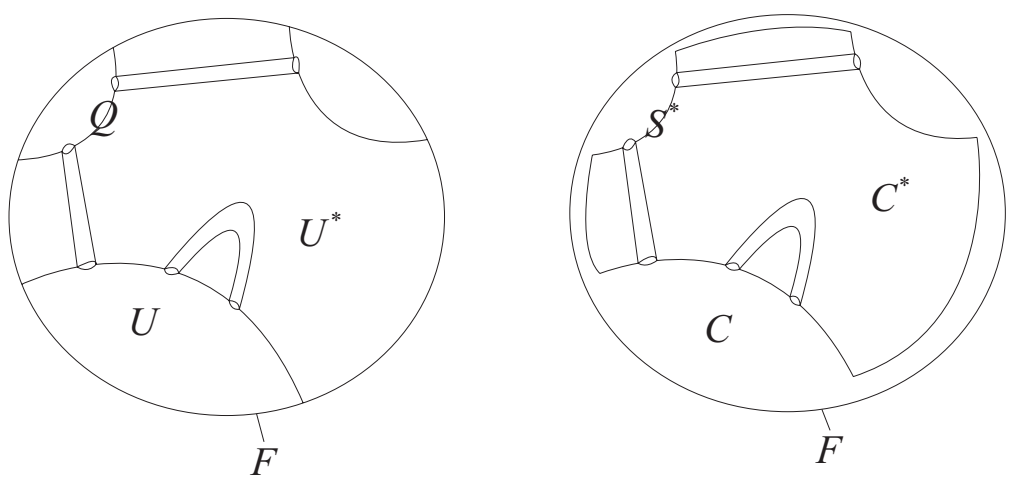

FigURE 3

Clearly, $2 g\left(S^{*}\right)=2-\chi\left(S^{*}\right) \leqslant 2-\chi(Q)-\chi(F) \leqslant 2-\chi\left(S \cap M_{2}\right)-\chi(F)$.

Note that we have proved that $-\chi\left(S \cap M_{2}\right) \leqslant 2 g\left(M_{2}\right)-2 g(F)-1$. Thus $2 g\left(S^{*}\right) \leqslant 2+2 g\left(M_{2}\right)-2 g(F)-1+2 g(F)-2=2 g\left(M_{2}\right)-1$. So $g\left(S^{*}\right)<g\left(M_{2}\right)$, a contradiction.

Proposition 3.2. Let $M$ be a compact, orientable 3-manifold and $F$ be an essential closed surface which cuts $M$ into $M_{1}$ and $M_{2}$. If $M_{i}$ has a Heegaard splitting $V_{i} \cup_{S_{i}} W_{i}$ with $D\left(S_{i}\right) \geqslant 2 g\left(M_{i}\right)+1, i=1,2$, then for any closed incompressible surface $F^{*}$ in $M$ with $g\left(F^{*}\right)<g\left(M_{1}\right)+g\left(M_{2}\right)$, we can isotope $F$ in $M$ such that $F \cap F^{*}=\emptyset$.

Proof. Since $F$ and $F^{*}$ are incompressible, we can isotope $F$ such that any component of $F \cap F^{*}$ is essential in both $F$ and $F^{*}$. Suppose $\left|F \cap F^{*}\right|$ is minimal. If $\left|F \cap F^{*}\right|>0$, then any component of $F^{*} \cap M_{i}$ is essential in $M_{i}$ since $\left|F \cap F^{*}\right|$ is minimal. 
So by Lemma 2.3, we have

$$
2-\chi\left(F^{*} \cap M_{1}\right) \geqslant D\left(S_{1}\right) \geqslant 2 g\left(M_{1}\right)+1
$$

and

$$
2-\chi\left(F^{*} \cap M_{2}\right) \geqslant D\left(S_{2}\right) \geqslant 2 g\left(M_{2}\right)+1 .
$$

Then

$$
4-\chi\left(F^{*} \cap M_{1}\right)-\chi\left(F^{*} \cap M_{2}\right) \geqslant 2 g\left(M_{1}\right)+2 g\left(M_{2}\right)+2
$$

i.e., $4-\chi\left(F^{*}\right) \geqslant 2 g\left(M_{1}\right)+2 g\left(M_{2}\right)+2$, so $g\left(F^{*}\right) \geqslant g\left(M_{1}\right)+g\left(M_{2}\right)$, a contradiction to the assumption.

Now we come to the proof of Theorem 1.1

Proof. By assumption, $M=M_{1} \cup_{F} M_{2}$ and $V_{i} \cup_{S_{i}} W_{i}$ is a Heegaard splitting with $D\left(S_{i}\right) \geqslant 2 g\left(M_{i}\right)+1, i=1,2$. Then by Lemma 2.4. $V_{i} \cup_{S_{i}} W_{i}$ is the unique minimal genus Heegaard splitting of $M_{i}$. Obviously, $M_{i}$ is irreducible, so $M$ is irreducible. We may assume that $F \subset \partial_{-} W_{1}, \partial_{-} V_{2}$. Let $V^{\prime} \cup_{S^{\prime}} W^{\prime}$ be an unstabilized Heegaard splitting of $M$ with

$$
g\left(S^{\prime}\right) \leqslant g\left(M_{1}\right)+g\left(M_{2}\right)-g(F) .
$$

Then by Theorem 3.1, $V^{\prime} \cup_{S^{\prime}} W^{\prime}$ is a weakly reducible and irreducible Heegaard splitting. By the result of [12], $V^{\prime} \cup_{S^{\prime}} W^{\prime}$ is an amalgamation of $n$ strongly irreducible Heegaard splittings $V^{\prime} \cup_{S^{\prime}} W^{\prime}=\left(V_{1}^{\prime} \cup_{S_{1}^{\prime}} W_{1}^{\prime}\right) \cup_{F_{1}^{\prime}}\left(V_{2}^{\prime} \cup_{S_{2}^{\prime}} W_{2}^{\prime}\right) \cup_{F_{2}^{\prime}} \cdots \cup_{F_{n-1}^{\prime}}$ $\left(V_{n}^{\prime} \cup_{S_{n}^{\prime}} W_{n}^{\prime}\right)$. Since $g\left(F_{i}^{\prime}\right)<g\left(S^{\prime}\right) \leqslant g\left(M_{1}\right)+g\left(M_{2}\right)-g(F)<g\left(M_{1}\right)+g\left(M_{2}\right)$, by Proposition [3.2, we can isotope $F$ so that $\left(\bigcup F_{i}^{\prime}\right) \cap F=\emptyset$. So $F$ lies in the non-trivial component $V_{j}^{*} \cup_{S_{j}^{*}} W_{j}^{*}$ of $V_{j}^{\prime} \cup_{S_{j}^{\prime}} W_{j}^{\prime}$, for some $1 \leqslant j \leqslant n$.

If $F$ is parallel to some component, say $F^{*}$, of $\bigcup F_{i}^{\prime}$, we amalgamate the Heegaard splitting sequence $V_{1}^{\prime} \cup_{S_{1}^{\prime}} W_{1}^{\prime}, V_{2}^{\prime} \cup_{S_{2}^{\prime}} W_{2}^{\prime}, \cdots, V_{n}^{\prime} \cup_{S_{n}^{\prime}} W_{n}^{\prime}$ along $\cup F_{i}^{\prime}-F^{*}$, and we obtain an unstabilized Heegaard splitting $V_{1}^{\prime *} \cup_{S_{1}^{\prime *}} W_{1}^{\prime *}$ of $M_{1}$ and an unstabilized Heegaard splitting $V_{2}^{\prime *} \cup_{S_{2}^{\prime *}} W_{2}^{\prime *}$ of $M_{2}$, such that $\partial_{-} W_{1}^{\prime *}=\partial_{-} V_{2}^{\prime *}=F^{*}$ and $g\left(S_{1}^{\prime *}\right)+g\left(S_{2}^{*}\right)-g(F)=g\left(S^{\prime}\right) \leqslant g\left(M_{1}\right)+g\left(M_{2}\right)-g(F)$. Then by Lemma 2.4, we have $g\left(S_{1}\right)=g\left(M_{1}\right) \leqslant g\left(S_{1}^{*}\right)$ and $g\left(S_{2}\right)=g\left(M_{2}\right) \leqslant g\left({S_{2}^{*}}^{*}\right)$, so $g\left(S_{1}\right)=g\left({S_{1}^{*}}^{*}\right)$ and $g\left(S_{2}\right)=g\left(S_{2}^{\prime *}\right)$. By Lemma 2.4. $V^{\prime} \cup_{S^{\prime}} W^{\prime}$ is the amalgamation of $V_{1} \cup_{S_{1}} W_{1}$ and $V_{2} \cup_{S_{2}} W_{2}$.

So we may assume that $F$ is not parallel to any component of $\bigcup F_{i}^{\prime}$. Then by Proposition 2.5. we may assume that any component of $F \cap S_{j}^{*}$ is essential in both $S_{j}^{*}$ and $F$, and at most one component of $S_{j}^{*} \backslash F$ is compressible in $M \backslash F$. Since $F$ is essential, $F \cap S_{j}^{*} \neq \emptyset$. We may assume that any component of $S_{j}^{*} \backslash F$ is incompressible in $M_{1}$. Then $S_{j}^{*} \cap M_{1}$ is essential in $M_{1}$. So $2-\chi\left(S_{j}^{*} \cap M_{1}\right) \geqslant$ $D\left(S_{1}\right) \geqslant 2 g\left(M_{1}\right)+1$.

If any component of $S_{j}^{*} \cap M_{2}$ is incompressible in $M_{2}$, then any component of $S_{j}^{*} \cap M_{2}$ is parallel to a subsurface of $F$. Since

$$
\begin{aligned}
2-\chi\left(S_{j}^{*} \cap M_{2}\right) & =2-\chi\left(S_{j}^{*}\right)+\chi\left(S_{j}^{*} \cap M_{1}\right) \\
& \leqslant 2 g\left(S^{\prime}\right)-2 g\left(M_{1}\right)+1 \\
& \leqslant 2 g\left(M_{2}\right)-2 g(F)+1 \\
& <D\left(S_{2}\right),
\end{aligned}
$$

we can isotope $S_{j}^{*}$ and $F$ such that $F \cap S_{j}^{*}=\emptyset$, a contradiction. 
Then we denote the compressible component of $S_{j}^{*} \cap M_{2}$ by $Q^{\prime}$ and assume that $Q^{\prime}$ is compressible in $V_{j}^{*}$. We compress $Q^{\prime}$ as much as possible in $V_{j}^{*}$ to obtain a surface $Q^{\prime *}$. Then any component of $Q^{\prime *}$ is incompressible in $V_{j}^{*} \cup_{S_{j}^{*}} W_{j}^{*}$ since $V_{j}^{*} \cup \cup_{S_{j}^{*}} W_{j}^{*}$ is strongly irreducible. Furthermore, $Q^{\prime *}$ is incompressible in $M_{2}$ since $\bigcup F_{i}^{\prime}$ is incompressible in $M . Q^{*}$ is parallel to the subsurfaces $F_{Q^{\prime *}}$ of $F$ since $2-\chi\left(Q^{*}\right) \leqslant 2-\chi\left(Q^{\prime}\right) \leqslant 2-\chi\left(S_{j}^{*} \cap M_{2}\right)<D\left(S_{2}\right)$. If one component of $F_{Q^{\prime *}}$ contains another component of $F_{Q^{\prime *}}$, then by the similar arguments of Theorem 3.1. $V_{j}^{\prime} \cup_{S_{j}^{\prime}} W_{j}^{\prime}$ is a non-trivial compression body. The Heegaard splitting $V_{j}^{\prime} \cup_{S_{j}^{\prime}} W_{j}^{\prime}$ is not strongly irreducible, a contradiction.

Any component of $F \cap V_{j}^{*}$ is incompressible in $V_{j}^{*}$. Then by Lemma 2.1 any component of $V_{j}^{*} \backslash F$ is a compression body. By the same reason as above, any component of $W_{j}^{*} \backslash F$ is a compression body. Let $U_{1}^{\prime}$ be the component of $V_{j}^{*} \backslash F$ containing $Q^{\prime}$ and $U_{2}^{\prime}$ be the component of $W_{j}^{*} \backslash F$ containing $Q^{\prime}$. We amalgamate the Heegaard splitting $V_{j}^{*} \cup_{S_{j}^{*}} W_{j}^{*}$ and the Heegaard splittings contained in $M_{2}$ of the Heegaard sequence $V_{1}^{\prime} \cup_{S_{1}^{\prime}} W_{1}^{\prime}, V_{2}^{\prime} \cup_{S_{2}^{\prime}} W_{2}^{\prime}, \cdots, V_{n}^{\prime} \cup_{S_{n}^{\prime}} W_{n}^{\prime}$ along the components contained in $M_{2}$ of $\bigcup F_{i}^{\prime}$ to obtain a Heegaard splitting $V_{3} \cup_{S_{3}} W_{3}$ such that the following conditions are satisfied:

(1) $V_{3} \cap M_{1}=V_{j}^{*} \cap M_{1}$ and $W_{3} \cap M_{1}=W_{j}^{*} \cap M_{1}$;

(2) $S_{3} \cap F=S_{j}^{*} \cap F$ and $S_{3} \cap M_{1}=S_{j}^{*} \cap M_{1}$;

(3) only one component of $S_{3} \cap M_{2}$ is compressible in $V_{3}$, denoted by $Q^{\prime \prime}$, and other incompressible components are just the components of $S_{j}^{*} \cap M_{2}$.

Then any component of $V_{3} \backslash F$ and $W_{3} \backslash F$ is a compression body. Let $U^{\prime}$ be the component of $V_{3} \backslash F$ which contains $Q^{\prime \prime}$ and $U^{\prime *}$ be the component of $W_{3} \backslash F$ which contains $Q^{\prime \prime}$. Then by the similar arguments of Theorem 3.1, the 3-manifold $U^{\prime} \cup_{Q^{\prime \prime}} U^{\prime *}$ is homeomorphic to $M_{2}$. Let $C^{\prime}=U^{\prime} \cup N\left(F \cap U^{\prime *}, U^{\prime *}\right)$ and $C^{\prime *}=$ $U^{\prime *} \backslash N\left(F \cap U^{\prime *}, U^{\prime *}\right)$. Then $C^{\prime}$ and $C^{\prime *}$ are compression bodies, $\partial_{+} C^{\prime}=\partial_{+} C^{*}=$ $S^{\prime *}$. So $C^{\prime} \cup_{S^{\prime *}} C^{\prime *}$ is a Heegaard splitting of $M_{2}$. We compare $g\left(S^{\prime *}\right)$ with $g\left(M_{2}\right)$.

Obviously, $g\left(S_{3}\right) \leqslant g\left(S^{\prime}\right) \leqslant g\left(M_{1}\right)+g\left(M_{2}\right)-g(F)$,

$$
\begin{gathered}
2-\chi\left(S_{3} \cap M_{1}\right)=2-\chi\left(S_{j}^{*} \cap M_{1}\right) \geqslant D\left(S_{1}\right) \geqslant 2 g\left(M_{1}\right)+1, \\
-\chi\left(S^{\prime *}\right) \leqslant-\chi\left(S_{3} \cap M_{2}\right)-\chi(F)=-\chi\left(S_{3}\right)+\chi\left(S_{j}^{*} \cap M_{1}\right)-\chi(F) .
\end{gathered}
$$

So

$2 g\left(S^{*}\right)-2 \leqslant 2 g\left(M_{1}\right)+2 g\left(M_{2}\right)-2 g(F)-2+\chi\left(S_{j}^{*} \cap M_{1}\right)+2 g(F)-2 \leqslant 2 g\left(M_{2}\right)-3$.

Thus we have $g\left(S^{*}\right)<g\left(M_{2}\right)$, a contradiction.

Hence $V^{\prime} \cup_{S^{\prime}} W^{\prime}$ is isotopic to the amalgamation of $V_{1} \cup_{S_{1}} W_{1}$ and $V_{2} \cup_{S_{2}}$ $W_{2}$ and $g(M)=g\left(S^{\prime}\right)=g\left(M_{1}\right)+g\left(M_{2}\right)-g(F)$. This completes the proof of Theorem 1.1.

\section{REFERENCES}

[1] D. Bachman and R. Derby-Talbot, Degeneration of Heegaard genus, a survey, arXiv:math.GT/0606383v3, preprint.

[2] D. Bachman, S. Schleimer and E. Sedgwick, Sweepouts of amalgamated 3-manifolds, Algebr. Geom. Topol. 6 (2006), 171-194. MR2199458(2006k:57057) 
[3] A. J. Casson and C. McA. Gordon, Reducing Heegaard splittings, Topology and Its Applications 27 (1987), 275-283. MR918537 (89c:57020)

[4] K. Harshorn, Heegaard splittings of Haken manifolds have bounded distance, Pacific J. Math. 204 (2002), 61-75. MR1905192 (2003a:57037)

[5] J. Hempel, 3-manifolds, Annals of Math. Studies, 86, Princeton University Press, 1976. MR0415619(54:3702)

[6] J. Hempel, 3-manifolds as viewed from the curve complex, Topology 40 (2001), 631-657. MR.1838999 (2002f:57044)

[7] W. Jaco, Lectures on three manifold topology, CBMS Regional Conference Series in Mathematics, 43, Amer. Math. Soc., 1980. MR565450 (81k:57009)

[8] T. Kobayashi, R. Qiu, Y. Rieck, and S. Wang, Separating incompressible surfaces and stabilizations of Heegaard splittings, Math. Proc. Camb. Phil. Soc. 137 (2004), 633-643. MR2103921 (2006c:57013)

[9] T. Kobayashi and R. Qiu, The amalgamation of high distance Heegaard splittings is always efficient, Math. Ann., Online: DOI 10.1007/s00208-008-0214-7.

[10] M. Lackenby, The Heegaard genus of amalgamated 3-manifolds, Geom. Dedicata 109 (2004), 139-145. MR2113191 (2005i:57021)

[11] T. Li, On the Heegaard splittings of amalgamated 3-manifolds, arXiv:math.GT/0701395, preprint.

[12] M. Scharlemann and A. Thompson, Thin position for 3-manifolds, Contemporary Math. 164, Amer. Math. Soc., 1994, 231-238. MR.1282766 (95e:57032)

[13] M. Scharlemann, Local detection of strongly irreducible Heegaard splittings, Topology and Its Applications 90 (1998), 135-147. MR1648310 (99h:57040)

[14] M. Scharlemann and M. Tomova, Alternate Heegaard genus bounds distance, Geom. Topol. 10 (2006), 593-617. MR 2224466 (2007b:57040)

[15] J. Schultens, Additivity of tunnel number for small knots, Comment. Math. Helv. 75 (2000), 353-367. MR1793793(2001i:57012)

[16] J. Schultens, The classification of Heegaard splittings for (compact orientable surfaces) $\times S^{1}$, Proc. London Math. Soc. 67 (1993), 425-448. MR1226608 (94d:57043)

[17] J. Souto, Distance in the curve complex and Heegaard genus, preprint.

Department of Mathematics, Harbin Institute of Technology, Harbin 150001, Heilonguiang Province, People's Republic of China

E-mail address: gqyang@hit.edu.cn

Department of Applied Mathematics, Dalian University of Technology, Dalian 116024, Liaoning Province, People's Republic of China

E-mail address: ffcclei@yahoo.com.cn 